\title{
Ostéonécrose des maxillaires dues aux bisphosphonates : mise au point
}

\section{Bisphosphonates-related jaw osteonecrosis: upcoming data}

\author{
SEMAAN ABI NAJM ${ }^{1}$, PHILIPPE LESCLOUS ${ }^{2}$, TOMMASO LOMBARDI' \\ IMEN BOUZOUITA ${ }^{1}$, JEAN-PIERRE CARREL ${ }^{1}$, JACKY SAMSON ${ }^{1}$
}

\section{RÉSUMÉ}

Les bisphosphonates sont des analogues structuraux des pyrophosphates inorganiques, prescrits essentiellement pour diminuer la résorption osseuse, donc l'ostéopénie, liée à des affections comme l'ostéoporose, la maladie de Paget, les dysplasies fibreuses ou les processus ostéolytiques rencontrés dans certaines affections malignes, en particulier le myélome multiple ou les métastases osseuses de tumeurs malignes solides (sein, prostate ...).

Les premiers cas d'ostéonécroses des maxillaires associées à la prise de bisphosphonates ont été rapportés en 2003 par Marx et coll. Depuis des centaines de cas ont été publiés et ce sujet a fait l'objet d'environ 600 publications.

La plupart de ces cas d'ostéonécrose sont survenus chez des patients traités pour des métastases osseuses de tumeurs solides ou pour un myélome multiple, avec le pamidronate ou le zolédronate administrés par voie I.V. Quelques cas ont été observés lors d'un traitement per os pour l'ostéoporose avec l'alendronate ou le risedronate mais l'évolution de l'ostéonécrose est bien différente. De multiples facteurs de risques peuvent jouer un rôle dans le développement de ces ostéonécroses ce qui rend difficile l'évaluation de leur prévalence et, de plus, leur fréquence augmente avec le temps.

Il existe encore des divergences dans les propositions ou les recommandations pour le traitement de ces ostéonécroses car on ne dispose pas encore de cohortes suffisantes avec un suivi prolongé. Med Buccale Chir Buccale 2007; $14: 5-18$.

mots clés: ostéonécrose, bisphosphonates.

\section{SUMMARY}

Bisphophonates are synthetic drugs analogs of inorganic pyrophosphate. These compounds are therapeutic agents used to inhibit bone resorption, so osteopenia, related to certain diseases like osteoporosis, Paget's disease of bone, fibrous dysplasia and effective antiresorptive agent for the treatment of diseases in which there is an increase in number or activity of osteoclasts, such as tumor associated osteolysis, multiple myeloma and bone metastasis in solid tumors ( breast, prostate...).

Marx et coll. in 2003 were the fist to report osteonecrosis of the jaws related to bisphosphonates treatment. Since then hundreds of cases where reported in over 600 publications.

\footnotetext{
1. Division de Stomatologie Chirurgie orale et Radiologie dento-maxillo-faciale Faculté de Médecine, Genève

2. Laboratoire sur la Réparation et les Remodelages orofaciaux EA 2496 Faculté de Chirurgie dentaire Université Paris Descartes
}

Demande de tirés à part:

Semaan Abi Najm Rue Barthélemmy-Menn 191205 Genève Suisse abns77@hotmail.com 
The majority of these patients received bisphosphonates for myeloma and bone metastasis. They were treated in particular by I.V bisphosphonates, pamidronate and zoledronate. A few cases have also been described in patients undergoing treatment with orally administrated bisphosphonates, alendronate and risedronate, for osteoporosis. Multiple risk factors are involved in bisphosphonate related jaw osteonecrosis which make frequency evaluation of this side effect difficult.

Multiple recommendations and protocols for treatment exists, but until today, cohort study and long term follow up are missed. Med Buccale Chir Buccale 2007; 14 : 5-18.

key words: osteonecrosis, bisphosphonates.

\section{GLOSSAIRE}

\begin{tabular}{|c|c|c|c|}
\hline AFSSAPS & agence française de sécurité & PGE 2 & prostaglandine E 2 \\
\hline & sanitaire des produits de santé & PTH & parathormone \\
\hline $\operatorname{aminoBP}(\mathrm{s})$ & aminobisphosphonate(s) & PTHrP & parathormone-related peptide \\
\hline $\mathrm{BMP}(\mathrm{s})$ & bone morphogenic protein(s) & RANK & receptor activator of nuclear \\
\hline $\mathrm{BP}(\mathrm{s})$ & bisphosphonate(s) & & kappa B \\
\hline FGF 2 & fibroblast growth factor 2 & RANKL & receptor activator of nuclear \\
\hline GTPase(s) & guanosine triphosphatase(s) & & kappa B ligand \\
\hline GVHD & graft versus-host disease & Rho, Ras, Rac & protéines humaines \\
\hline HNG-CoA & $\begin{array}{l}\text { hydroxyl methyl } \\
\text { glutaryl-coenzyme A }\end{array}$ & SAPHO & $\begin{array}{l}\text { synovite-acné-pustulose- } \\
\text { hyperostose-ostéite }\end{array}$ \\
\hline IGF & insulin-like growth factor & SERM & selective estrogen receptor \\
\hline M-CSF & mononuclear phagocyte & $\mathrm{FB}$ & $\begin{array}{l}\text { modulators } \\
\text { nor } \beta\end{array}$ \\
\hline OPG & ostéoprotégérine & & \\
\hline
\end{tabular}

Les bisphosphonates (BPs) sont des analogues structuraux des pyrophosphates inorganiques, prescrits essentiellement pour diminuer la résorption osseuse, donc pour prévenir l'ostéopénie, observée dans certaines affections telles que l'ostéoporose, la maladie de Paget, les dysplasies fibreuses, la nécrose aseptique de la hanche, l'osteogenesis imperfecta ou les processus ostéolytiques rencontrés dans certaines affections malignes comme le myélome multiple ou les métastases osseuses de tumeurs malignes solides (sein, prostate ...). Ces molécules existent sous deux formes chimiques: les BPs sans groupement amine et les BPs avec un groupement amine ou aminoBPs qui correspondent aux produits de dernière génération ${ }^{[57-61]}$. Ils constituent un progrès important dans la prise en charge des affections citées ci-dessus. Ils sont souvent administrés au long cours et leurs effets indésirables ont été initialement considérés comme mineurs si la posologie était respectée.

Les BPs de dernière génération (aminoBPs) peuvent induire une ostéonécrose des maxillaires décrite pour la première fois en 2003 [35]. En quelques années, plusieurs centaines de cas ont été rapportés mais un nombre plus important de cas a très probablement été observé sans être répertorié. L'ostéonécrose se traduit par un retard de cicatrisation après une extraction dentaire, par la perte spontanée d'une dent ou parfois par l'apparition, sans cause apparente, d'une exposition osseuse dans une région édentée. La plupart des cas d'ostéonécrose sont survenus chez des patients traités pour des métastases osseuses 
de tumeurs solides ou pour un myélome multiple, avec le pamidronate ou le zoledronate administrés par voie IV [36,67], mais quelques cas ont été observés lors d'un traitement per os pour l'ostéoporose avec l'alendronate ou le risédronate [59]. La survenue d'une ostéonécrose chez les patients traités par des BPs sans groupement amine reste exceptionelle [2-46].

Ces lésions sont probablement à rapprocher des nécroses observées chez des travailleurs de l'industrie des allumettes exposés au phosphore blanc, qui ont été décrites pour la première fois il y a presque deux siècles [25], puis dans l'industrie des feux d'artifice et de l'armement. Elle se traduisait par une exposition osseuse, toujours intra-buccale, assez souvent douloureuse, s'accompagnant ou non d'une surinfection, dont l'évolution spontanée pouvait aboutir à des délabrements bucco-faciaux importants. Elle a disparu avec l'arrêt de l'utilisation du phosphore pour la fabrication des allumettes et dans l'industrie des feux d'artifice et de l'armement. Quelques cas ont été encore observés après la seconde guerre mondiale dans l'industrie de l'armement.

Plusieurs théories éthiopathogéniques ont été proposées pour expliquer la relation exacte entre ces ostéonécroses et le traitement par BPs, mais aucune n'est totalement satisfaisante. Après une revue de la littérature, les trente premiers cas traités dans la Division de Stomatologie, Chirurgie orale et Radiologie dento-maxillo-faciale de l'Ecole de Médecine dentaire de Genève seront présentés dans un autre article (Ostéonécrose des maxillaires due aux bisphosphonates : présentation de 30 cas), à paraitre dans le prochain numéro de $\mathrm{MBCB}$.

\section{REMANIEMENT OSSEUX PHYSIOLOGIQUE}

Le tissu osseux est une structure dynamique en perpétuel remaniement. On distingue des phénomènes de modelage et de remodelage. Le modelage qui permet la mise en forme des os, intervient principalement au cours de la croissance. Le remodelage permet le renouvellement du tissu osseux, en remplaçant l'os âgé ou altéré par un os jeune [49].
Pour le remodelage (résorption puis formation), l'unité fonctionnelle est constituée par deux types de cellules, les ostéoclastes et les ostéoblastes. Les ostéoclastes, cellules géantes multinucléées, d'origine macrophagique, sont responsables de la résorption osseuse : ils détruisent l'os âgé ou altéré en quelques jours. Les ostéoblastes sont des cellules mésenchymateuses qui synthétisent en quelques semaines une nouvelle matrice osseuse. II existe un équilibre dans le remodelage qui permet l'homéostasie de la masse osseuse au cours de la vie adulte. Un troisième type cellulaire, les ostéocytes, dont la fonction a été peu étudiée, joue un rôle important dans le transport de substances organiques et inorganiques à l'intérieur de l'os. Le cycle de remodelage osseux comporte plusieurs phases qui se déroulent sur environ 150 jours (Fig. 1) :

- Phase de repos : la surface de l'os est recouverte de cellules bordantes qui servent de couche de protection pour l'os sous-jacent.

- Phase d'activation des ostéoclastes : sous l'action des facteurs ostéorésorbants (PTH, vitamine D3 et PGE 2) [19,67], les cellules bordantes libérent l'accès aux ostéoclastes qui rentrent en contact avec la matrice osseuse. Les ostéoblastes sont indispensables à la mise en place du programme de différenciation des précurseurs ostéoclastiques en pré-ostéoclastes, puis en ostéoclastes et enfin en ostéoclates actifs, grâce à l'action du M-CSF et de deux autres molécules, I'OPG et le RANKL qui se fixe sur son récepteur ostéoclastique, le RANK [33]. - Phase de résorption ostéoclastique : la résorption osseuse ostéoclasique comprend plusieurs étapes : la prolifération des précurseurs des ostéoclastes, la différenciation des précurseurs en ostéoclastes mononucléés, leur fusion pour donner les ostéoclastes multinucléés, la formation de la zone claire et de la bordure plissée lors de leur activation [9].

- Phase de transition : quand l'ostéoclaste a creusé une lacune, il disparait par apoptose et il est remplacé par des macrophages qui régularisent le fond de la lacune.

- Phase de synthèse : lorsque la résorption osseuse est terminée, les cellules ostéoprogénitrices présentes à la surface de la matrice érodée, au fond de la lacune (ou ligne cémentante), se divi- médecine

buccale

chirurgie

buccale

VOL. $14, \mathrm{~N}^{\circ} 1$ 2008

page 7 
médecine

buccale

chirurgie

buccale

VOL. $14, \mathrm{~N}^{\circ} 1$ 2008

page 8

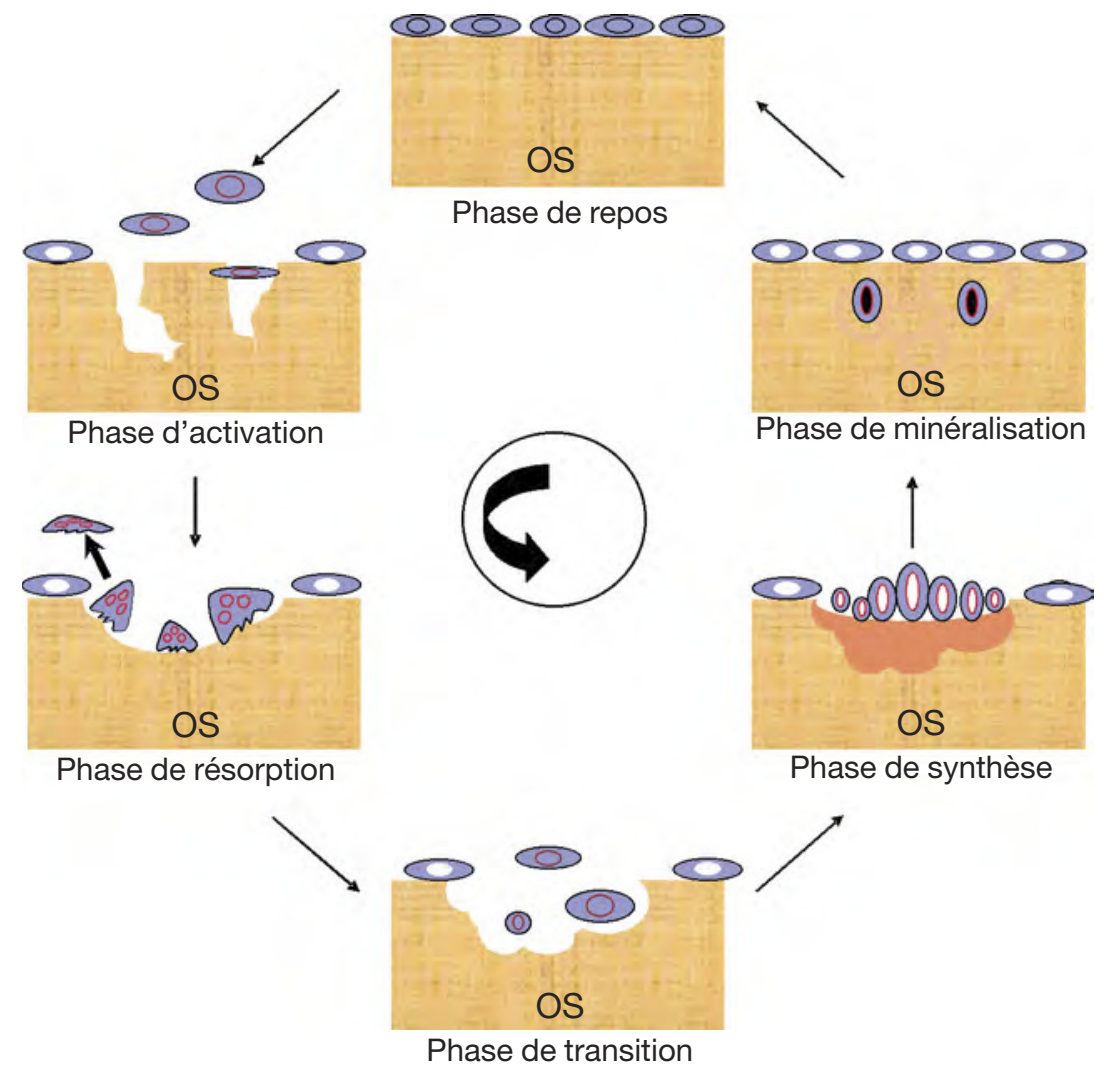

$\begin{array}{ll}\infty \text { Cellule bordante } & \text { Ostéoblaste } \\ \infty \text { Ostéoclaste } & \text { Cellule mononuclée }\end{array}$

Figure 1 : Différentes phases du remodelage osseux [10]. Different phases of bone remodeling ${ }^{[10]}$.

sent et se différencient en ostéoblastes. Ces ostéoblastes synthétisent une nouvelle matrice non minéralisée (tissu ostéoïde) qui comble la lacune. Plusieurs hormones (oestrogènes, androgènes et vitamine $D$ ) et de nombreux facteurs de croissance sécrétés par les ostéoclastes (FGF 2, TGFB, IGF et BMPs) stimulent la production de la matrice osseuse et jouent un rôle essentiel dans l'ostéogénèse. A la fin de la phase de formation, les ostéoblastes disparaissent par apoptose ou se retrouvent inclus dans la matrice ostéoïde sous forme d'ostéocytes.

- Phase de minéralisation : la minéralisation commence sur le front de minéralisation. Les phos- phatases alcalines osseuses initieraient la minéralisation du tissu ostéoïde en influençant la concentration locale en ions calcium et phosphates. L'ostéocalcine augmente la concentration locale du calcium extracellulaire et de la vitamine D3 ; pour mémoire, cette dernière permet l'absorption intestinale du calcium et favorise sa fixation sur l'os.

- Phase de repos : les ostéoblastes qui n'ont pas disparu par apoptose, se transforment en cellules bordantes.

Après la fin de la croissance, l'os continue à subir un remodelage permanent. La masse de l'os spongieux représente $20 \%$ du squelette avec un 
turnover de $80 \%$, celle de l'os cortical $80 \%$ avec un turnover de $20 \%$ [10]. On considère qu'environ $10 \%$ du squelette se renouvelle chaque année, ce qui signifie que la totalité du squelette est renouvelée tous les dix ans [10].

L'altération du processus de remodelage et/ou du couplage entre résorption et formation de l'os entraîne des modifications importantes de l'architecture et de la densité osseuses qui peuvent aboutir à long terme à une ostéoporose, une ostéomalacie ou une maladie ostéocondensante.

\section{BISPHOSPHONATES}

\section{Historique}

Jadis appelés diphosphonates, les BPs sont connus depuis la fin du 19 e siècle : la première synthèse des BPs a été réalisée en Allemagne en 1865 et ils ont été principalement utilisés comme inhibiteurs de la corrosion dans l'industrie textile et dans celle de l'huile [23]. Depuis une quarantaine d'années, les BPs sont utilisés dans le traitement de certaines pathologies du métabolisme osseux. Au début des années 60, Neuman et Fleisch réalisent une étude sur le mécanisme des calcifications induites par le collagène et ils démontrent que le plasma et l'urine de l'homme contiennent des inhibiteurs de la calcification. Fleisch et coll. [23] mettent en évidence un pyrophosphate inorganique, un polyphosphate naturel, dans le sérum et l'urine qui peut prévenir la calcification et régulariser la minéralisation osseuse en se fixant sur les cristaux d'hydroxyapatite. En biochimie, l'importance des phosphatases est bien connue : elles entrent dans la composition des acides nucléiques, des os et des autres tissus minéralisés comme la dentine ou le cément, et elles jouent un rôle primordial dans les réactions biochimiques intervenant dans le transfert d'énergie. La recherche médicale s'est intéressée à l'inhibition de la calcification des dérivés phosphatés, surtout pour prévenir les calcifications hétérotopiques dans les tissus mous. Cependant, l'utilisation du pyrophosphate pour prévenir la formation des plaques d'athérome, la calcification des valves cardiaques et la formation des lithiases urinaires a été un échec. Fleisch a remarqué que les pyrophosphates étaient rapidement hydrolysés par une enzyme, la pyrophosphatase, largement présente dans les différents tissus de l'organisme [23,in 61]. Cette découverte a permis d'orienter la recherche vers des substances ayant des propriétés physico-chimiques similaires aux pyrophosphates mais résistant à l'hydrolyse enzymatique, ce qui a conduit à la découverte des BPs [29]. Comme les pyrophosphates, les BPs possèdent une grande affinité pour la composante osseuse minérale.

\section{Bisphopshonates commercialisés}

Comme les BPs, analogues structuraux des pyrophosphates inorganiques, résistent à l'hydrolyse enzymatique, ils sont devenus les produits de référence surtout pour le traitement des affections ostéolytiques, bénignes ou malignes, grâce à leurs propriétés antirésorbantes. L'atome central d'oxygène de la liaison $\mathrm{P}-\mathrm{O}-\mathrm{P}$ du pyrophosphate inorganique est remplacé par un atome de carbone (liaison P-C-P) rendant la molécule de BPs résistante à l'hydrolyse enzymatique ${ }^{[61]}$ (Fig. 2).<smiles>O=P(O)(O)OP(=O)(O)O</smiles>

Pyrophosphate inorganique

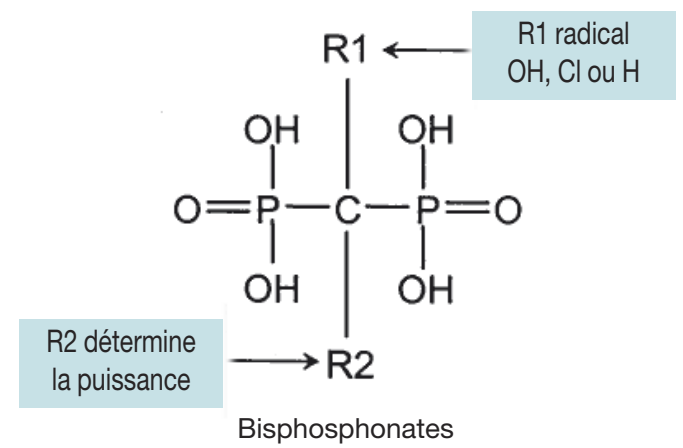

médecine

buccale

chirurgie buccale

VOL. $14, \mathrm{~N}^{\circ} 1$ 2008

page 9

Figure 2 : Structure du pyrophosphate inorganique et des BPs : noter l'analogie entre leur structure. Structure of inorganic pyrophosphate and BPs: note the analogy of the two structures. 
Tableau 1 : Structure moléculaire et puissance des BPs commercialisés [10]. Molecular structure and potency of commercialized BPs [10].

\begin{tabular}{|c|c|c|c|c|}
\hline $\mathrm{DCl}$ & Nom commercial & R1 & $\mathbf{R 2}$ & Puissance \\
\hline Etidronate & Didronel ${ }^{\circledR}$ & $\mathrm{OH}$ & $\mathrm{CH}_{3}$ & 1 \\
\hline Clodronate & Clastoban ${ }^{\circledR}$ & $\mathrm{Cl}$ & $\mathrm{Cl}$ & 10 \\
\hline Tiludronate & Skélid ${ }^{\circledR}$ & $\mathrm{H}$ & $\mathrm{S}-\mathrm{Cl}$ & 10 \\
\hline Pamitronate & Arédia $^{\circledR}$ & $\mathrm{OH}$ & $\mathrm{CH}_{2}-\mathrm{CH}_{2}-\mathrm{NH}_{2}$ & 100 \\
\hline Alendronate & Fosamax $^{\circledR}$ & $\mathrm{OH}$ & $\mathrm{CH}_{2}-\mathrm{CH}_{2}-\mathrm{CH}_{2}-\mathrm{NH}_{2}$ & 1000 \\
\hline Risédronate & Actonel ${ }^{\circledR}$ & $\mathrm{OH}$ & $\mathrm{CH}_{2}-\square^{\mathrm{N}}$ & 5000 \\
\hline Ibandronate & $\begin{array}{c}\text { Bondronat }^{\circledR} \\
\text { Boniva }^{\circledR}\end{array}$ & $\mathrm{OH}$ & $\mathrm{CH}_{2}-\mathrm{CH}_{2}-\mathrm{N}-\mathrm{C}_{5} \mathrm{H}_{11}$ & 10000 \\
\hline Zoledronate & Zometa $^{\circledR}$ & $\mathrm{OH}$ & $\mathrm{CH}_{2}-\mathrm{N} \Longrightarrow \mathrm{N}$ & 20000 \\
\hline
\end{tabular}

VOL. $14, N^{\circ} 1$ 2008 page 10
La liaison P-C-P confère aux BPs une grande affinité pour le tissu osseux et on obtient de nombreux produits en modifiant les radicaux $\mathrm{R} 1$ et $\mathrm{R} 2$ liés à l'atome de carbone central. Le radical $\mathrm{R} 1$ est le plus souvent le radical hydroxyl $(\mathrm{OH})$ car il permet d'obtenir la meilleure adhésion sur le tissu osseux minéral. Le radical $\mathrm{R} 2$ détermine la puissance d'action des $\mathrm{BPs}$; il ne comporte pas d'atome d'azote (BPs simples) ou il comporte un atome d'azote (aminoBPs). Les aminoBPs ont une activité sur les ostéoclastes 10 à 20000 fois plus importante que celle de l'étidronate, produit de première génération, qui est considéré comme la molécule référence ${ }^{[61]}$ (Tab. 1).

Chaque BPs a une structure chimique et physicochimique et des caractéristiques biologiques spécifiques : leurs propriétés biologiques, leur activité, leur cinétique pharmacologique et leur toxicité sont donc différentes, ce qui rend difficile toute extrapolation des résultats d'une structure à l'autre [61]

\section{MODE D'ACTION DES BISPHOSPHONATES}

\section{Effets biologiques des bisphosphonates}

Les BPs ont deux effets biologiques fondamentaux : l'inhibition de la calcification dans les tissus mous et l'inhibition de la résorption osseuse.

\section{Inhibition de la calcification dans les tissus mous ${ }^{[23, \text { in } 61]}$}

Les BPs préviennent les calcifications ectopiques in vivo grâce à leur propriété régulatrice sur le métabolisme du calcium endogène (Fleisch, 1995). Plusieurs études expérimentales animales (Francis et coll., 1969 ; Fleisch et coll., 1970 ; Casey et coll., 1972) et humaines (Bassett et coll., 1969 ; Cram et coll., 1971 ; Russell et coll., 1972) ont prouvé l'efficacité des BPs, administrés per os ou par voie IV, dans la prévention des calcifications extra-osseuses non seulement par leur action sur le tissu osseux minéral mais aussi par leurs effets sur le cholestérol, l'élastine et le collagène des parois artérielles. Depuis trois décennies, les BPs ont prouvé leurs effets dans le traitement de certaines affections calcifiantes comme la myosite ossifiante (Bassett et coll., 1969 ; Russell et coll., 1972) et la calcinose (Cram et coll., 1971).

Quelques études ont confirmé leur action inhibitrice dans l'athérosclérose. Meradji et coll. ont été les premiers à démontrer en 1978 l'effet bénéfique des BPs dans les calcifications artérielles : en se déposant dans les parois artérielles, ils empêchent l'accumulation des lipoprotéines dans les macrophages, or cette accumulation représente la première phase du processus d'athério- 
sclérose. Récemment les BPs ont été utilisés dans le traitement des calcifications intracérébrales ${ }^{[30]}$.

\section{Inhibition de la résorption osseuse}

Les études in vitro et in vivo ont montré que l'inhibition de la résorption osseuse constitue le principal effet des BPs $[23,29,61]$. Cet effet s'observe aussi bien chez les sujets sains que chez les sujets atteints d'une affection ostéolytique. Les BPs se fixent de façon sélective sur la structure cristalline de l'hydroxyapatite de la phase minérale du tissu osseux : ils sont absorbés par les ostéoclastes ce qui perturbe leur fonctionnement et induit leur apoptose ${ }^{[41]}$ (Fig. 3). Les BPs ne sont pas métabolisés : environ la moitié de la dose se fixe sur l'os, l'autre moitié est éliminée par le rein, sans modification de leur structure.

L'activité inhibitrice sur les ostéoclastes dépend de la structure du radical $R 2$ et se fait par deux voies métaboliques distinctes. Les BPs qui ne possèdent pas de groupement amine (BPs simples) dans le radical $\mathrm{R} 2$ (étidronate, clodronate, tiludronate), se substituent à l'ATP pour former un métabolite cytotoxique pour les cellules. En revanche, les aminoBPs (pamidronate, risédronate, ibandronate,
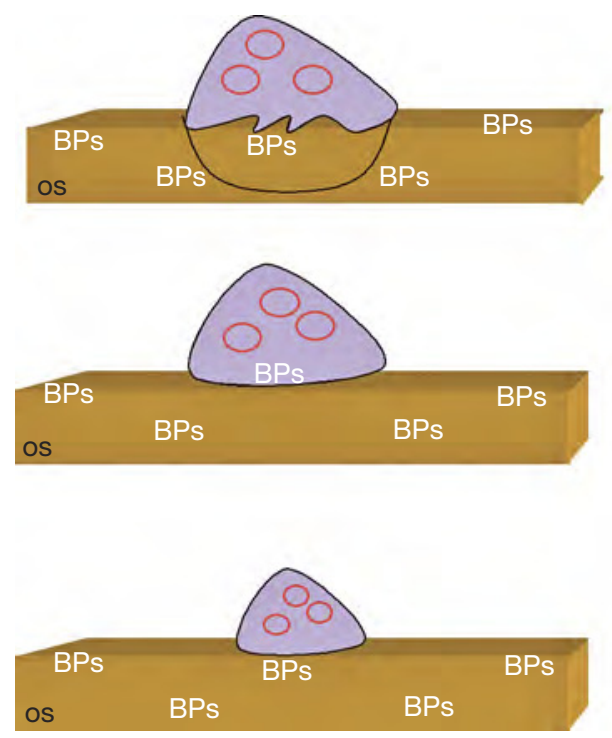

BPs : molécule de BPs alendronate, zoledronate) interfèrent avec la voie du mévalonate en inhibant la prénylation des GTPases (Rho, Ras, Rac.... ${ }^{[22,61]}$ qui jouent un rôle essentiel dans les mécanismes de signalisation intracellulaire (Fig. 4) ; cette action entraine l'inhibition de l'activité de résorption des ostéoclastes [16].

\section{Autres effets}

Quelques molécules de BPs ont une activité antiangiogénique ${ }^{[12,13,16,68]}$ : le zoledronate et l'ibandronate auraient la capacité d'inhiber l'angiogénèse ou la néovascularisation, processus nécessaire à la croissance tumorale et à la dissémination métastatique ${ }^{[16]}$.

\section{Effets indésirables}

Ils sont bien connus et pour la plupart mineurs et transitoires [14]. L'insuffisance rénale aiguë constitue l'effet indésirable le plus grave mais elle peut être prévenue par une administration IV lente. Avec la majorité des BPs, on observe un syndrome pseudo-grippal (élévation temporaire de la température, frissons, sensation de fatigue, arthralgies et myalgies) qui régresse habituellement en 48 heures. Des manifestations digestives sont régulièrement rapportées : nausées, vomissements, diarrhées,

médecine

buccale

chirurgie

buccale

VOL. $14, \mathrm{~N}^{\circ} 1$ 2008

page 11
Absorption des bisphosphonates par les ostéoclastes

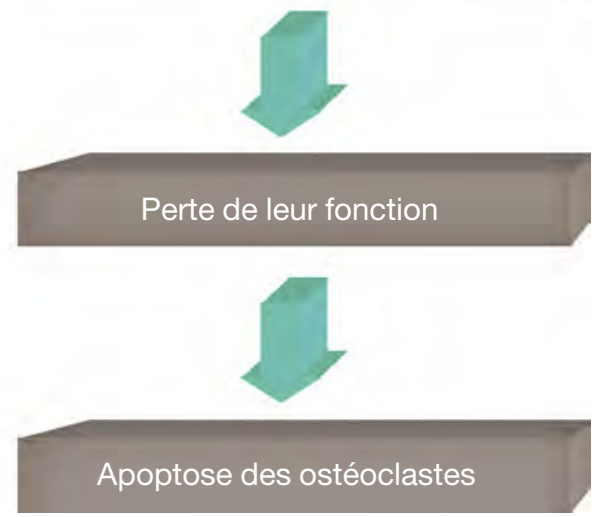

Ostéoclaste

Figure 3 : Effets des BPs sur les ostéoclastes [22]. Effect of BPs on osteoclasts [22]. 


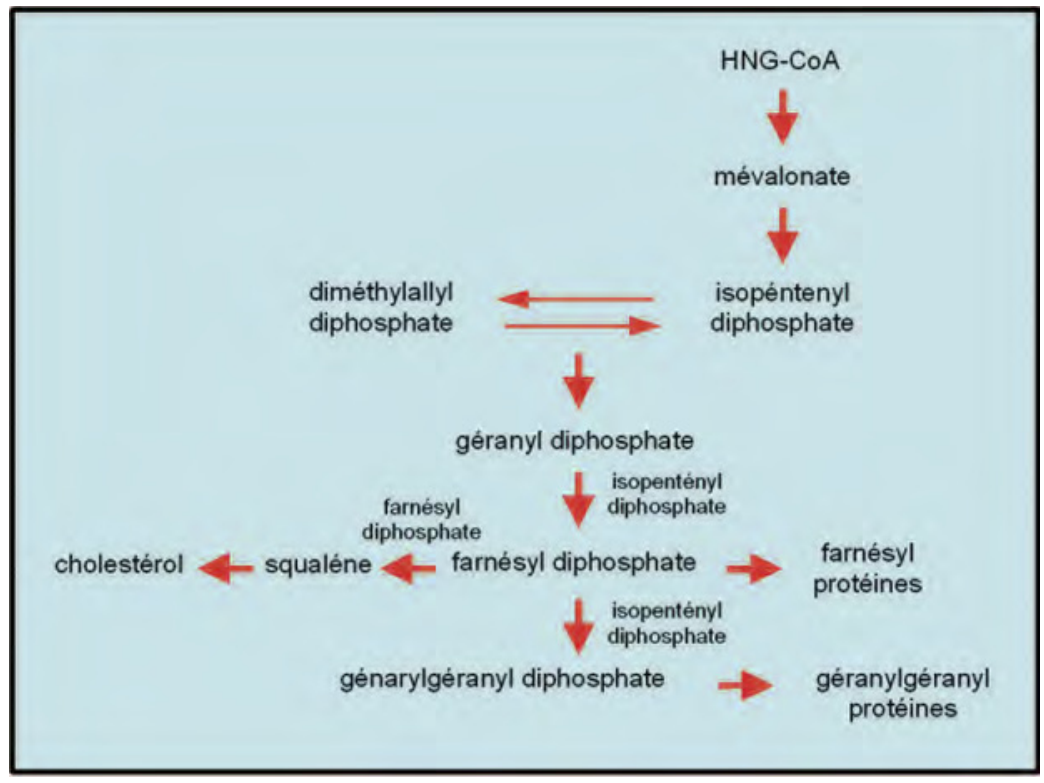

médecine buccale chirurgie buccale

VOL. $14, \mathrm{~N}^{\circ} 1$ 2008

page 12
Figure 4 : Voie du mévalonate [22]. Mevalonate pathway [22].

douleurs abdominales, œsophagite, ulcérations buccales. Des ulcères gastriques et une sensation de sécheresse buccale sont parfois observés. La formule sanguine est souvent perturbée de façon transitoire : anémie, thrombocytopénie, leucopénie, parfois pancytopénie. Les effets indésirables neurologiques se traduisent par des céphalées, des vertiges, des sensations d'hypoesthésie ou à l'inverse d'hyperesthésie, et une dysgueusie. De rares cas d'hypersensibilité immédiate et d'érythème polymorphe ont été rapportés. L'étidronate induit parfois une altération de la réponse immunitaire qui se traduit par la réactivation d'un herpès secondaire ou le développement d'un zona. De rares manifestations oculaires, à type d'uvéite et de conjonctivite, peuvent être observées. Les anomalies métaboliques, principalement une hypocalcémie, sont le plus souvent asymptomatiques ; parfois elles se traduisent par des crampes musculaires ou des épisodes de tétanie.

En 2003, les premiers cas d'ostéonécrose des maxillaires en relation avec la prise d'aminoBPs ont été rapportés [35]. Cet effet indésirable semble concerner seulement un faible pourcentage des patients traités, mais le recul est sans doute insuffisant pour évaluer la fréquence réelle de cette complication.

\section{INDICATIONS DES BISPHOSPHONATES}

En trois décennies, les indications des BPs se sont progressivement élargies. Après avoir été initialement utilisés dans la maladie osseuse de Paget, ils sont maintenant prescrits pour le traitement et la prévention de l'ostéoporose, pour le traitement de l'hypercalcémie maligne et des tumeurs osseuses d'origine hématologique ou métastatique qui s'accompagnent d'une ostéolyse et, plus récemment, pour le traitement de la nécrose aseptique de la hanche, du syndrome $\mathrm{SAPHO}$, de certaines dysplasies fibreuses des os et de l'osteogenesis imperfecta ${ }^{[4,5]}$.

\section{Prévention et traitement de l'ostéopo- rose \\ Définition de l'ostéoporose}

L'ostéoporose est une affection qui s'accompagne d'une augmentation du risque de fractures osseuses secondaires à une diminution de la masse osseuse. Cette affection constitue un problème mondial de santé publique ${ }^{[10]}$. Les fractures caractéristiques de l'ostéoporose intéressent les vertèbres, sous la forme de «tassements " vertébraux, l'extrémité supérieure du fémur et l'extrémité distale de l'avant-bras (AFSSAPS 2006) ${ }^{[52] .}$ 


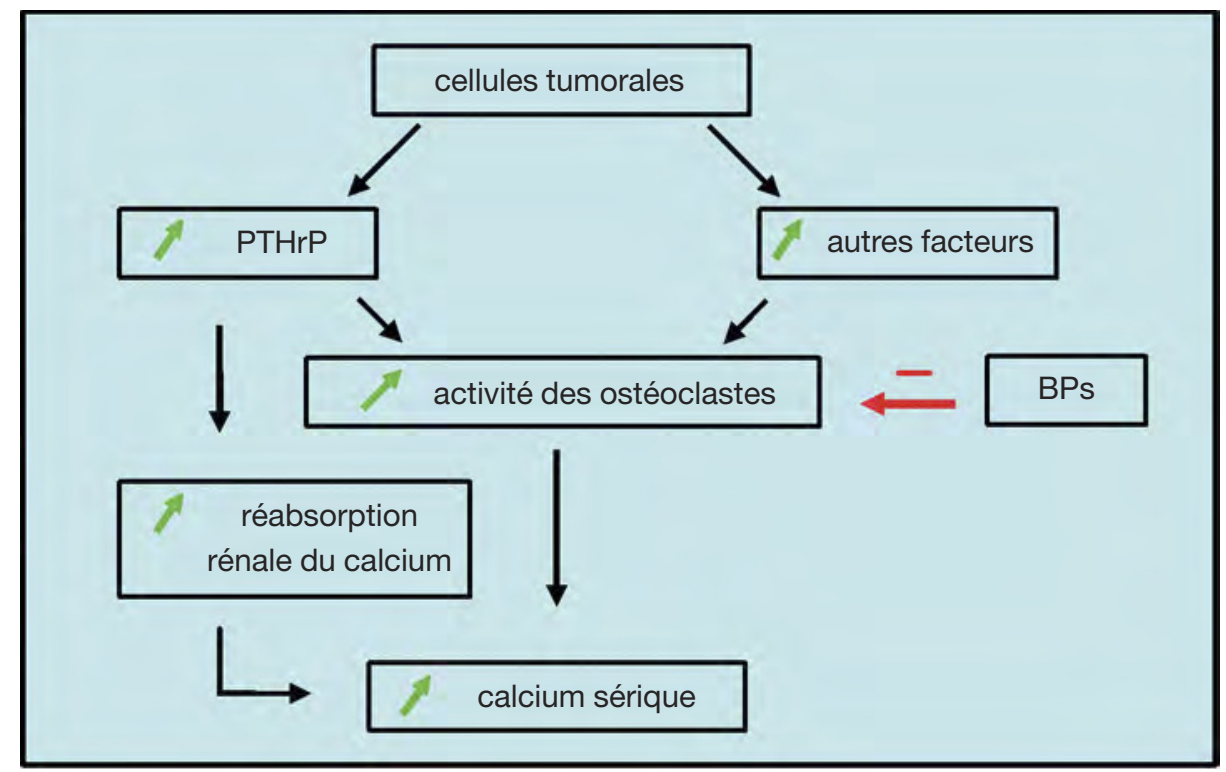

Figure 5 : Pathogénie de l'hypercalcémie tumorale et mode d'action des BPs [12].

Hypercalcemia of malignant disease pathogenesis and mechanism of action of BPs [12].

Elle est extrêmement fréquente puisqu'elle toucherait $30 \%$ des femmes après 50 ans et $70 \%$ à partir de 80 ans. La physiopathologie de l'ostéoporose apparait de plus en plus complexe. Les facteurs nutritionnels (calcium et vitamine $D$ ) et les facteurs hormonaux (œstrogènes et PTH) restent les principaux éléments impliqués dans la perte osseuse. On distingue deux formes cliniques d'ostéoporose : l'ostéoporose post-ménopausique qui est la plus fréquente, les ostéoporoses dites secondaires, parmi lesquelles celle induite par la corticothérapie. Dans l'ostéoporose, le traitement a pour but de prévenir la perte osseuse, donc des fractures (accord professionnel, AFSSAPS 2006) [52]. Plusieurs études, réalisées sur des cohortes importantes, ont permis de quantifier les bénéfices et les risques du traitement de l'ostéoporose, et d'en préciser les indications ${ }^{[1,56]}$.

\section{Traitements disponibles}

Depuis une décennie, la prise en charge de l'ostéoporose, surtout chez la femme ménopausée, a évolué avec l'apparition de nouveaux traitements : les BPs, les SERM et les substances anabolisantes stimulant la formation osseuse (la PTH et le ranélate de strontium). Récemment une nouvelle approche thérapeutique de l'ostéoporose a été proposée. II s'agit d'un anticorps monoclonal, le dénozumab, qui se lie au RANKL, protéine essentielle pour la différenciation, l'activation et la survie de l'ostéoclaste.

L'alendronate et le risédronate représentent le traitement le plus prescrit pour l'ostéoporose. En diminuant le remaniement osseux, ils entraînent une augmentation de la densité minérale osseuse. Plusieurs études cliniques, avec un suivi sur 10 ans, ont montré l'efficacité et la tolérance de ces molécules dans le traitement de l'ostéoporose à long terme ${ }^{[56]}$. Selon les recommandations de I'AFSSAPS, l'alendronate et le risédronate constituent le traitement de référence pour l'ostéoporose post-ménopausique et des études récentes ont montré que le traitement par l'alendronate ou le risédronate devait être poursuivi au moins 4 ans (AFSSAPS 2006). Au-delà, la poursuite du traitement repose sur une réévaluation individuelle du risque fracturaire ${ }^{[52]}$.

\section{Traitement de l'hypercalcémie maligne}

Les BPs constituent un progrès thérapeutique important pour le traitement de certaines pathologies osseuses, en particulier pour celui de l'hypercalcémie maligne et des affections osseuses métastatiques. Les deux principales causes d'hy- médecine

buccale chirurgie buccale

VOL. $14, \mathrm{~N}^{\circ} 1$ 2008

page 13 
médecine

buccale

chirurgie

buccale

VOL. $14, \mathrm{~N}^{\circ} 1$ 2008

page 14 percalcémie sont l'hypercalcémie maligne et l'hyperparathyroïdie primaire. L'hypercalcémie maligne est le plus souvent observée dans le cancer du sein et le cancer du poumon [12-62]. Les BPs ont supplanté tous les autres moyens thérapeutiques utilisés pour traiter l'hypercalcémie maligne (Fig. 5) : ils normalisent la calcémie chez plus de $90 \%$ des patients, mais ils sont moins efficaces lorsque l'hypercalcémie récidive. Ils réduisent de façon importante (de 20 à $60 \%$ selon les auteurs) [21] la fréquence des manifestations liées à l'atteinte osseuse, en diminuant les douleurs, les fractures pathologiques, les compressions radiculaires ou médullaires et les épisodes d'hypercalcémie maligne. Dans le cancer du sein, il est recommandé de commencer le traitement par BPs dès que la maladie métastatique osseuse devient symptomatique et même, pour certains auteurs, dès que le diagnostic de métastases osseuses est établi. Pour le myélome multiple où l'ostéolyse joue un rôle déterminant dans la progression de la maladie, il est recommandé de commencer le traitement par les BPs au stade III, stade avancé du myélome caractérisé par un des critères suivants : hémoglobine $<8.5 \mathrm{~g} / \mathrm{dl}$, calcémie $>120 \mathrm{mg} / \mathrm{l}$, lésions osseuses multiples, taux élevé d'immunoglobines.

\section{Autres indications (maladie de Paget, dysplasie fibreuse des os...)}

Les indications des BPs se sont élargies ces dernières années à d'autres affections osseuses bénignes comme la maladie de Paget [55], le syndrome SAPHO [5,64], l'osteogenesis imperfecta, certaines dysplasies fibreuses des os, l'ostéomyélite chronique des maxillaires... La maladie osseuse de Paget et la dysplasie fibreuse des os sont des affections caractérisées par des anomalies du remodelage osseux, systémiques ou focales, où l'on observe une augmentation de la résorption ostéoclastique et du remodelage osseux. Dans la maladie osseuse de Paget, les BPs entraînent une diminution des douleurs, une régression des zones ostéolytiques, un arrêt de la progression de la maladie avec reconstitution d'un os histologiquement normal. Dans la dysplasie fibreuse des os, les BPs diminuent les douleurs et entraînent un comblement partiel des foyers ostéolytiques et un épaississement des corticales [40].

Les BPs représentent un progrès majeur dans le traitement de nombreuses affections osseuses comportant des anomalies de la résorption osseuse.

\section{OSTÉONÉCROSE DES MAXILLAIRES ET BISPHOSPHONATES}

\section{Epidémiologie}

La prévalence de ces ostéonécroses est difficile à évaluer car leur fréquence augmente avec le temps. II semble qu'elle soit différente selon la molécule prescrite, la posologie et la durée de traitement. Les données de littérature concernent principalement la prévalence de l'ostéonécrose des maxillaires chez des patients traités pour une affection maligne. En 2004, une enquête réalisée par internet a été menée par l'International Myeloma Foundation pour évaluer les facteurs de risque de l'ostéonécrose [20]. Sur les 1203 cas répertoriés, 904 cas étaient traités pour un myélome multiple et 299 pour un cancer de sein. Dans les 904 cas de myélome multiple, 62 (7\%) présentaient une ostéonécrose et 54 (6\%) des lésions suspectes ; $71 \%$ étaient traités par le zoledronate et $29 \%$ par le pamidronate seuls. Dans les 299 cas de cancer du sein, 13 (4\%) patientes présentaient une ostéonécrose et 23 (8\%) des lésions suspectes. Sur une période de 36 mois, la prévalence est de $10 \%$ chez les patients traités par le zoledronate et de $4 \%$ chez ceux traités par le pamidronate seuls. En 2005, Bamias ${ }^{[8]}$ a réalisé une étude prospective sur 252 patients traités par BPs pour des métastases ostéolytiques entre janvier 1997 et décembre 2003, avec un bilan effectué jusqu'en février 2005 : 17 (6,7\%) patients ont développé une ostéonécrose des maxillaires. Sur ces 17 cas, $11(9,9 \%)$ cas ont été retrouvés chez 111 patients traités pour un myélome multiple, 2 (2,9\%) cas chez 70 patientes traitées pour un cancer du sein, $3(6,5 \%)$ cas chez 46 patients traités pour un cancer de la prostate et $1(4 \%)$ cas chez 25 patients traités pour un autre néoplasme. Tous les patients présentant une ostéonécrose avaient eu un traitement par le zoledronate seul (7 patients), ou après pamidronate ( 9 patients), ou avant iban- 
dronate (1 patient). En 2006, Zervas et coll. [70] ont publié une étude concernant 303 patients ayant un myélome multiple : les 28 (11\%) cas d'ostéonécrose sont retrouvés uniquement chez les 254 patients traités par BPs.

Dans la revue de la littérature réalisée par Woo et coll. [67], la prévalence des ostéonécroses varie de $6 \%$ à $10 \%$ pour les patients traités pour une affection maligne. En 2007, Mavrokoki et coll. [37] publient une étude sur la fréquence des ostéonécroses pour les patients sous zoledronate ou pamidronate pour une affection maligne et chez les patients traités par l'alendronate pour une ostéoporose. La fréquence de survenue des ostéonécroses dans le premier groupe varie de $0,88 \%$ à $1,15 \%$ et elle augmente après un acte chirurgical $(6,67 \%$ à $9,1 \%)$. Pour le groupe traité par l'alendronate, la fréquence est moins importante : elle varie de $0,01 \%$ à $0,04 \%$ en l'absence d'acte chirurgical et de $0,09 \%$ à 0,34 \% après une extraction dentaire.

\section{Etiopathogénie}

L'incorporation osseuse des BPs est proportionnelle à l'intensité du remodelage osseux au moment de leur utilisation, leur concentration est donc plus élevée dans les zones de croissance, les sites osseux en cours de cicatrisation, les sites tumoraux et ceux qui présente naturellement un taux de remodelage physiologique plus important comme l'os alvéolaire des maxillaires [38]. C'est probablement la raison pour laquelle les ostéonécroses associées aux BPs concernent presque toujours les maxillaires et exceptionnellement d'autres sites [6,51]. Toutefois, le mécanisme étiopathogénique de l'ostéonécrose n'est pas clairement élucidé. Plusieurs hypothèses ont été proposées.

L'ostéonécrose serait due à une diminution du remodelage osseux physiologique induit par les BPs $[36,60]$ (théorie de l'hypocellularité). Cette diminution du remodelage pourrait aussi être secondaire à une diminution de la vascularisation osseuse. Elle est d'autant plus importante que le BPs est puissant : les BPs ont la propriété d'inhiber l'activité des ostéoclastes et de provoquer leur apoptose ce qui diminue la résorption osseuse et donc perturbe le remaniement osseux normal [26]. La vascularisation intra-osseuse serait également affectée par l'effet anti-angiogénique de certaines molécules de BPs comme le zoledronate et le pamidronate ${ }^{[11,68]}$ (théorie de l'hypovascularisation). Un traitement fortement dosé pourrait conduire à un os "gelé » dont le remodelage est inhibé de façon trop importante ${ }^{[47]}$. Cet effet est surtout observé avec les aminoBPs puissants comme le pamidronate et le zoledronate ${ }^{[12]}$, mais il peut être aussi observé avec l'alendronate [48]. Cet os inerte a perdu toute capacité de réparation des microfractures physiologiques induites par la fonction (théorie des microfractures) ${ }^{[34,66]}$.

Dans la cavité buccale, les maxillaires sont soumis, lors de la fonction (mastication) ${ }^{[48]}$ et des parafonctions (bruxisme), à un stress régulier qui stimulerait l'activité de remodelage entraînant une incorporation plus importante des BPs. Cette accumulation de BPs pourrait induire une réduction drastique du remodelage favorisant la nécrose osseuse. Dès lors la vascularisation de la muqueuse sus-jacente est réduite et tout traumatisme mineur pourrait entraîner une exposition de l'os nécrosé sous-jacent. La colonisation secondaire par la flore buccale de l'os nécrosé exposé peut être responsable de douleurs ou d'accidents infectieux souvent révélateurs de l'ostéonécrose (théorie infectieuse) [32]. Une autre hypothèse suggère que l'ostéonécrose pourrait se développer à partir de la muqueuse [54]. La diminution du turn over osseux par les BPs aurait un effet « toxique » sur la muqueuse buccale : ceci expliquerait l'apparition d'une déhiscence de la muqueuse, la contamination bactérienne de l'os à partir du milieu entrainant le développement d'une ostéonécrose et l'absence d'évolution favorable de la cicatrisation (théorie toxique). Enfin certains facteurs génétiques prédisposants pourraient favoriser le développement de l'ostéonécrose [32].

\section{Aspects clinique et radiologique}

Cliniquement, l'atteinte osseuse intrabuccale ressemble plus à une ostéonécrose qu'à une ostéomyélite : exposition d'un os blanc jaunâtre ou blanc grisâtre, qui reste de consistance dur, sans formation de séquestre dans les délais habituels et sans aucune tendance à la guérison spontanée ${ }^{[3,67]}$.

Dans $60 \%$ des cas rapportés dans la littérature, l'ostéonécrose apparait souvent secondaire à un acte chirurgical intéressant l'os alvéolaire : extrac- médecine

buccale

chirurgie

buccale

VOL. $14, \mathrm{~N}^{\circ} 1$ 2008

page 15 
médecine buccale chirurgie buccale

VOL. $14, \mathrm{~N}^{\circ} 1$ 2008

page 16 tion dentaire, mise en place d'un implant dentaire, chirurgie parodontale [67] ; dans les autres cas, elle semble spontanée. Pour Marx et coll., $39 \%$ des cas d'ostéonécrose spontanée sont localisés sur des exostoses ou des reliefs osseux où la muqueuse est fine et facilement traumatisée [36].

Le bilan radiologique comporte un orthopantomogramme, et parfois un examen tomodensitométrique dans les ostéonécroses étendues. Les signes radiologiques sont souvent absents au début de l'évolution : l'ostéonécrose est visible cliniquement alors qu'il n'y pas de signe d'atteinte osseuse sur les radiographies conventionnelles (radiographie rétro-alvéolaire, orthopantomogramme). En fait, les BPs en diminuant le remodelage osseux augmentent la densité radiologique de l'os dans un premier temps ${ }^{[47]}$. Cet aspect perdure en général plusieurs mois dans les maxillaires malgré le développement d'une ostéonécrose, et ce n'est qu'après une longue évolution que l'on observe une image ostéolytique mal définie, avec parfois l'apparition d'un séquestre qui se déminéralise lentement.

\section{Facteurs de risque Molécules}

La composition de la molécule de BPs prescrite joue un rôle prépondérant pour le développement de l'ostéonécrose. Cette complication est décrite exclusivement avec les aminoBPs $[2,3,7,8,15,24,28,36$, 42,43,44,45,50,67]. Actuellement, cinq molécules ont été mentionnées dans le développement des ostéonécroses : le zoledronate, le pamidronate, l'alendronate, le risédronate et l'ibandronate (Tab. 2).

$94 \%$ des cas d'ostéonécrose rapportés sont retrouvés chez des patients traités par des BPs administrés par voie parentérale, pamidronate et/ou zoledronate $[2,3,31,39,67]$. Le risque de développement d'une ostéonécrose est plus élevé avec le zoledronate qu'avec le pamidronate seul ou l'association pamidronate puis zoledronate ${ }^{[6,8,20]}$. Cette différence s'explique probablement par la puissance antirésorbante inférieure du pamidronate par rapport à celle du zoledronate ${ }^{[58]}$.

\section{Dose cumulée}

La dose cumulée qui représente la dose totale de BPs administrée avant l'apparition de l'ostéonécrose, constitue sans doute le facteur prédisposant le plus important pour le développement de l'ostéonécrose. II n'y a qu'une seule étude prospective qui a évalué cette relation : elle a clairement montré que l'incidence de l'ostéonécrose des maxillaires est corrélée à la dose cumulée des BPs ${ }^{[8]}$ (Fig. 6).

Tableau 2 : Dénomination commune internationale, dénomination commerciale en Suisse, posologie et voie d'administration des BPs associés aux ostéonécroses des maxillaires. ( $\mathrm{j}$; jour ; sem : semaine).

Generic name, brand name, posologie and administration way of BPs associated with osteonecrosis of the jaws. (j: day ; sem: week).

\begin{tabular}{|c|c|}
\hline BPs & $\begin{array}{l}\text { Posologie et voie } \\
\text { d'administration }\end{array}$ \\
\hline $\begin{array}{l}\text { Zoledronate } \\
\left.\text { (Zometa }^{\circledR}\right)\end{array}$ & $\begin{array}{l}4 \mathrm{mg} / \mathrm{mois} \\
\text { parentérale }\end{array}$ \\
\hline $\begin{array}{l}\text { Pamidronate } \\
\text { (Arédia } 囚)\end{array}$ & $\begin{array}{c}30,60 \text { ou } \\
90 \mathrm{mg} / \mathrm{mois} \\
\text { parentérale }\end{array}$ \\
\hline $\begin{array}{c}\text { Ibandronate } \\
\left(\text { Boniva }^{\circledR}\right) \\
\left(\text { Bondronate }^{\circledR}\right)\end{array}$ & $\begin{array}{c}2 \text { ou } 6 \text { mg/mois } \\
\text { parentérale }\end{array}$ \\
\hline
\end{tabular}

\begin{tabular}{|c|c|}
\hline BPs & $\begin{array}{c}\text { Posologie et voie } \\
\text { d'administration }\end{array}$ \\
\hline Alendronate & $\begin{array}{c}5 \text { ou } 10 \mathrm{mg} / \mathrm{j} \\
35 \text { ou } 70 \mathrm{mg} / \mathrm{sem} \\
\text { per os }\end{array}$ \\
\hline (Fosamax $\left.{ }^{\circledR}\right)$ & $5 \mathrm{mg} / \mathrm{j}$ \\
\hline Risédronate & $35 \mathrm{mg} / \mathrm{sem}$ \\
\hline per os
\end{tabular}




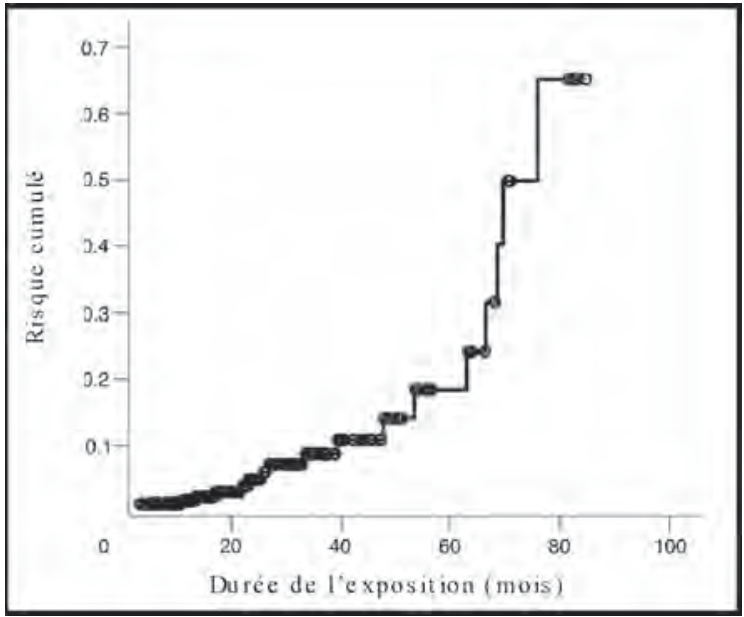

Figure 6 : Augmentation du risque de développement d'une ostéonécrose en fonction

de la dose cumulée, donc avec la durée du traitement par BPs chez 252 patients ${ }^{[8]}$.

Cumulative hazard of developing osteonecrosis of the jaw from the date of treatment among 252 patients treated with BPS ${ }^{[8]}$.

Tableau 3 : Biodisponibilité des différentes molécules de BPs [17].

Biodisponibility of different molecules of BPs [17].

\begin{tabular}{|c|c|}
\hline BPs & $\begin{array}{c}\text { Biodisponibilité } \\
\text { (taux) }\end{array}$ \\
\hline $\begin{array}{l}\text { Zoledronate } \\
\text { Zometa® (IV) }\end{array}$ & $55-77 \%$ \\
\hline $\begin{array}{l}\text { Pamidronate } \\
\text { Arédia } \otimes(I V)\end{array}$ & $45-80 \%$ \\
\hline $\begin{array}{c}\text { lbandronate } \\
\text { Bondronate } ® \text { (IV) } \\
\text { Bonviva } ® \text { (IV- per os) }\end{array}$ & $40 \%$ \\
\hline $\begin{array}{c}\text { Alendronate } \\
\text { Fosamax® (per os) }\end{array}$ & $0,64 \%$ \\
\hline 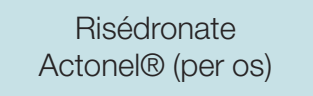 & $<1 \%$ \\
\hline $\begin{array}{c}\text { Ibandronate } \\
\text { Bondronate }{ }^{\circledR} \text { (per os) }\end{array}$ & $0,6 \%$ \\
\hline
\end{tabular}

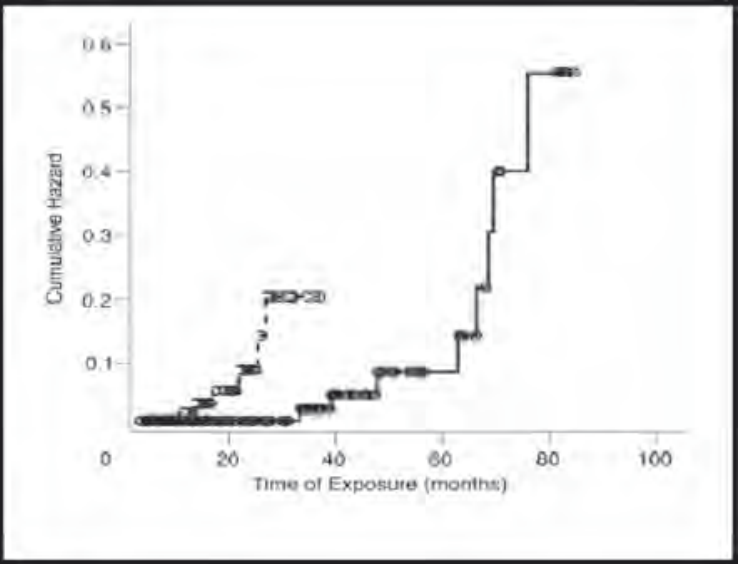

Figure 7 : Augmentation du risque cumulé pour le développement d'une ostéonécrose pour les patients traités par le zoledronate, le pamidronate ou l'association pamidronate-zoledronate en fonction de la durée du traitement ${ }^{[8]}$.

Cumulative hazard of developing osteonecrosis of the jaw according to treatment with zoledronic acid or pamidronate \pm zoledronic acid according to time of exposure ${ }^{[8]}$.

Cette même étude a démontré que la survenue de l'ostéonécrose est plus précoce pour les patients traités par le zoledronate que pour ceux traités par le pamidronate ou l'association pamidronate puis zoledronate (Fig. 7).

Le délai entre la première prise de BPs et l'apparition d'une ostéonécrose des maxillaires est très variable. Ce délai dépend de la molécule (voie d'administration, puissance et biodisponibilité), de la fréquence d'administration, donc de la dose cumulée. Par exemple, l'alendronate est dix fois plus puissant que le pamidronate (Tab. 1) mais sa biodisponibilité est très faible per os (Tab. 3 ) : la biodisponibilité de l'alendronate per os est beaucoup plus faible que celle de pamidronate administré par voie IV. La dose cumulée ne reflète qu'imparfaitement la quantité fixée sur l'os. On devrait plutôt se référer à la dose absorbée qui correspond à la dose cumulée corrigée par le taux de biodisponibilité de la molécule. médecine buccale chirurgie buccale

VOL. $14, \mathrm{~N}^{\circ} 1$ 2008

page 17 
médecine

buccale

chirurgie

buccale

VOL. $14, \mathrm{~N}^{\circ} 1$ 2008

page 18
Rôle des traumatismes et du site anatomique

Le développement d'une ostéonécrose des maxillaires se traduit initialement, assez souvent, par un retard de cicatrisation après une intervention intéressant l'os alvéolaire, en général après une extraction dentaire [67], mais la cause ayant motivé l'extraction dentaire est très rarement précisée. II est aussi important de remarquer qu'il existe dans certaines études un pourcentage non négligeable (37 \%) d'ostéonécroses qui ne sont pas corrélées à un traumatisme local (ostéonécroses spontanées) [36-39].

La mandibule constitue la localisation préférentielle : $65 \%$ des cas intéressent la mandibule, $26 \%$ des cas le maxillaire et $9 \%$ des cas présentent une double localisation maxillaire et mandibulaire [66]. Les secteurs postérieurs mandibulaires semblent être plus concernés que les régions symphysaire et parasymphysaire mandibulaires ${ }^{\left[{ }^{[}\right]}$.

\section{Traitements proposés}

Les propositions et les recommandations pour le traitement des ostéonécroses dues aux bisphosphonates sont multiples $[2,18,36,44,53,59]$ mais on ne dispose pas encore de cohortes suffisantes avec un suivi prolongé.

En juin 2004, un groupe d'experts a publié des recommandations pour le traitement des ostéonécroses sur aminoBPs. Les patients étaient divisés en trois catégories ${ }^{[59]}$ :

- Groupe 1 : les patients qui vont commencer un traitement par aminoBPs,

- Groupe 2 : les patients traités par aminoBPs sans ostéonécrose des maxillaires,

- Groupe 3 : les patients avec ostéonécrose des maxillaires.

Les recommandations pour chaque groupe étaient les suivantes :

Groupe 1 : mise en état de la cavité buccale, élimination des foyers infectieux, instauration d'une bonne hygiène bucco-dentaire. II n'est probablement pas nécessaire de retarder le début du traitement par aminoBPs si le traitement d'assainissement buccodentaire peut être terminé en 1 à 2 mois.

Groupe 2 et 3 : réhabilitation par traitements conservateurs (traitement de racine, surfaçage radiculaire ...), éviter les interventions chirurgi- cales. Devant une ostéonécrose, élimination à minima de l'os exposé, rinçage buccal (chlorhexidine, peroxyde d'hydrogène), antibiothérapie, analgésie, couverture de la zone exposée par une plaque de résine molle.

Marx et coll. ont proposé un protocole de traitement spécifique du stade clinique de l'ostéonécrose [36].

- patient à risque, sans exposition osseuse :

- élimination des foyers infectieux,

- si possible, arrêt des aminoBPs IV pendant 2 ou 3 mois, c'est-à-dire pendant le temps nécessaire pour obtenir un état bucco-dentaire satisfaisant.

- patient présentant une exposition osseuse asymptomatique :

- rinçage buccal 3 fois/jour avec de la chlorhexidine à 0,12 \%,

- pas de traitement systémique.

- patient présentant une exposition osseuse accompagnée d'une infection et de douleurs :

- rinçage buccal 3 fois/jour avec de la chlorhexidine à 0,12\%,

- antibiothérapie (pénicilline, doxycycline, levofloxacine ou érythromycine).

- patient n'ayant pas eu d'évolution favorable sous antibiothérapie :

- antibiothérapie (pénicilline, doxycycline, levofloxacine ou érythromycine),

- résection osseuse (alvéolectomie ou résection plus large).

Récemment, I'AFSSAPS a publié des recommandations sur la prise en charge bucco-dentaire des patients traités par bisphosphonates [53]. Ces recommandations diffèrent peu de celles présentées ci-dessus : on retrouve la même approche et les mêmes groupes. Pour les patients atteints d'une ostéonécrose avérée, il est conseillé de réaliser le traitement en milieu hospitalier. Les traitements chirurgicaux ne doivent pas être agressifs et les reconstructions par greffes libres ou pédiculées sont déconseillées. Il est nécessaire d'informer le médecin prescripteur de cette complication. La poursuite du traitement par BPs doit être décidée au cas par cas par le médecin prescripteur.

La bibliographie complète paraîtra avec le second article (Ostéonécrose des maxillaires due aux bisphosphonates : présentations de 30 cas). 